\title{
The ethics of phase I trials of biologic agents
}

\author{
Stephen B Hanauer
}

In addition to having several other responsibilities, I lead a clinical research group and have designed and implemented, and have been a principal investigator for, numerous investigatorinitiated and industry-sponsored clinical trials. For the most part my group has participated in phase II or III clinical trials, but we have performed a few phase I studies. I was recently asked to participate in a phase I clinical trial of a new biologic agent for IBD, but have declined for what I believe to be ethical reasons.

Phase I clinical trials usually occur after pilot studies have been completed in animals to deduce the potential mechanisms of action, pharmacology and toxicology of a drug. As such, they are generally the first stage of drug testing in humans. Phase I trials are required and designed to assess the safety, tolerability, pharmacokinetics and pharmacodynamics of a drug in humans. However, unlike phase II or III trials, phase I trials are not intended to demonstrate or confirm clinical efficacy. This latter point is extremely important when patients are recruited, as the concept of equipoise regarding the potential benefit of a therapy is not applicable - there are no preliminary data (and no rationale) available to be able to present an informed risk-benefit statement when obtaining the consent of a patient to enter the trial. Therefore, phase I clinical trials are usually performed in normal, healthy individuals or terminally ill patients. Often, healthy controls are paid to participate in phase I trials in contrast to the volunteers recruited for phase II or III trials

Even for phase II trials (and some phase III trials) I am afraid that some patients have been 'burned' by their participation-and not from unexpected toxicity. Instead, with the number of trials of biologic agents increasing, numerous volunteer patients have been immunized against a biologic by their early exposure to it, and have
... . with the

number of trials

of biologic

agents

increasing,

numerous

volunteer

patients

have been

immunized

against a

biologic by their

early exposure

to it...

SB Hanauer is

Editor-in-Chief

of Nature

Clinical Practice

Gastroenterology

\& Hepatology.

Competing interests

The author has declared

associations with the

following companies:

Abbott, Bristol Myer Squibb,

Centocor, Genentech,

GlaxoSmithKline and UCB.

See the article online for full

details of the relationships.

www.nature.com/clinicalpractice doi:10.1038/ncpgasthep1259 subsequently developed hypersensitivity reactions and loss of response when re-exposed to the same drug once it has been approved for marketing.

I learned a lesson with infliximab, the first biologic agent approved for the treatment of Crohn's disease. In the early (phase II) studies of infliximab, patients in the treatment groups were exposed to a single infusion or a series of three infusions of infliximab (N Engl J Med [1997] 337: 1029-1035; N Engl J Med [1999] 340: 1398-1405). Although such studies demonstrated the remarkable efficacy of infliximab and led to FDA approval, once the studies were completed, patients were unable to obtain any further doses of infliximab until it was marketed 6-18 months later. When patients who responded so splendidly (and after a relatively short duration) were re-exposed to marketed infliximab, approximately $25 \%$ developed severe delayed hypersensitivity reactions that precluded subsequent dosing (Gastroenterology [2003] 124: 11401150). Ultimately, these patients were unable to benefit from the drug that they volunteered to test because of the development of antiinfliximab antibodies. Furthermore, nearly a decade passed before a second, alternative anti-TNF agent - adalimumab - became available. These folk were clearly canaries in a mine shaft...and they got the shaft!

Consequently, I now have serious concerns regarding enrolling patients into phase I, or even phase II, trials of agents that have the potential to induce the development of antibodies, unless it is clearly stated during 'informed consent' that participating in the clinical trial could preclude them from gaining any benefit from the experimental therapy, even if it is eventually proven to be effective and approved for marketing. 